\title{
A Study of Ad-Hoc Wireless Networks: Various Issues in Architectures and Protocols
}

\author{
Sushmita Kopekar \\ Department of Electronics \& \\ Telecommunications Engineering, G. H. Raisoni \\ College of Engineering (An Autonomous \\ Institution), Nagpur Maharashtra, India
}

\author{
Amresh Kumar \\ Department of Computer Technology, \\ Rajiv Gandhi College of Engineering \& \\ Research, Nagpur, Maharashtra, India
}

\begin{abstract}
A wireless ad-hoc network is a collection of nodes which are self-organizing and self-administering in nature. All nodes are connected by wireless links and are free to move randomly. Thus, the network topology keeps on changing rapidly and erratically. These kinds of networks are very flexible and don't need any existing infrastructure. In order establish communication within the network and to discover the routes between the nodes a routing protocol is used so that messages may be delivered in a timely manner. The primary objective of an ad hoc network routing protocol is accurate and efficient route establishment between a pair of nodes. Route construction should be done with a minimum of overhead and minimum consumption of bandwidth. The major challenge in these kinds of networks is finding a path between the communication end points of nodes that are mobile. Therefore, these kinds of networks are also called multi-hop ad-hoc networks. This paper is based on study of ad-hoc wireless networks and it's related various issues in architectures and protocols. This paper provides an overview of various issues and challenges in various protocols.
\end{abstract}

\section{Keywords}

Wireless network, Ad hoc Network, Multi-hop, Routing protocols, Infrastructureless

\section{INTRODUCTION}

Ad-hoc wireless networks [1] [2] [6] comprise of sets of nodes connected by wireless links that form arbitrary wireless network topologies without the use of any centralized access point. Ad-hoc wireless networks are inherently self-forming, self-arranging and self-administering. The nodes are free to move arbitrarily and organize themselves at random. Thus topology of the network may alter quickly and unpredictably. The biggest challenge in these kinds of networks is finding the path between the communication end points of nodes that are movable. Because of restricted transmission range of wireless interfaces, the communication traffic has to be extended over a number of intermediate nodes to facilitate the communication among two nodes. Thus, these kinds of networks are also called as multi-hop ad-hoc networks. Every node acts as both, a host and as a router. Another limitation associated with wireless devices is the power constraint of the nodes i.e. each node has only limited battery power which should be used judiciously for the node to survive longer.

The principle behind ad hoc network is multi-hop relaying. The use of ad hoc voice communication was used in many ancient/tribal societies with the help of a string of repeaters of drums, trumpets, or horns. In 1970, Norman Abramson and his fellow researchers at the University of Hawaii invented the ALOHAnet, an innovative communication system for linking together the universities of the Hawaiian Islands. ALOHAnet utilized single-hop wireless packet switching and a multiple access solution for sharing a single channel. The success and novelty of ALOHAnet triggered widespread interest in different directions of computer communication, including the work that led to the development of Ethernet and the packet radio network (PRNET) project sponsored by the defense advanced research projects agency (DARPA). DARPA extended the work on multi-hop wireless networks through the survivable radio networks (SURAN) project that aimed at providing ad hoc networking with small, low-cost, low-power devices with efficiency protocols and improved scalability and survivability. Bluetooth SIG aims at delivering a universal solution for connectivity among heterogeneous devices. Hence it is one of the first commercial recognition of ad hoc wireless networking. Bluetooth standardizes the single-hop point-to-point wireless link that helps in exchanging voice or data, and formation of piconets that are formed by a group of nodes in a smaller geographical region where every node can reach every other node in the group within a single-hop. Multiple piconets can form a scatternet, which necessitates the use of multi-hop routing protocols. Even with all the promises that are offered ad hoc wireless networks, successful commercial deployment requires realistic solutions to different problems, including support for QoS provisioning, concurrent applications, pricing, cooperative operation, energy-efficient relaying, load balancing and maintenance for multicast traffic.

This paper is based on study of ad-hoc wireless networks and related issues in various architectures and protocols for ad hoc networks. Thus the paper provides an overview of various issues and challenges in various protocols.

The remainder of the paper is organized as follows. The next section presents a discussion the basic concepts of Cellular and Ad-Hoc Wireless Networks. Another section discusses Applications of Ad-Hoc Wireless Networks, while a later section describes Issues in Ad-Hoc Wireless Networks. The paper then presents the various issues and challenges in designing of various ad-hoc wireless networks protocols and other ad-hoc wireless networks area. Finally the paper concludes with the introduction of some recent and open area for research and investigation.

\section{CELLULAR AND AD-HOC WIRELESS NETWORKS}

The current cellular wireless networks [1] [6] are classified as the infrastructure dependent networks. The path setup for a call between two nodes is completed through the base station. Ad hoc wireless networks are defined as the category of wireless networks that utilize muti-hop radio relaying and arc capable of operating without the support of any fixed infrastructure (hence 
they are also called as infrastructureless networks). The absence of any central coordinator or base station makes routing a complex one compared to cellular networks. In a cellular network the presence of base stations simplifies routing and resource management as the routing decisions are made in a centralized manner with more information about the destination node. But in an ad hoc wireless network, the routing and resource management are done in a distributed manner in which all nodes coordinate to facilitate communication among the nodes. This requires each node to be more intelligent so that it can function both as a network host for transmitting and receiving data and also as a network router for routing packets. That is why the mobile nodes in ad hoc wireless networks are more complex than that of cellular networks.

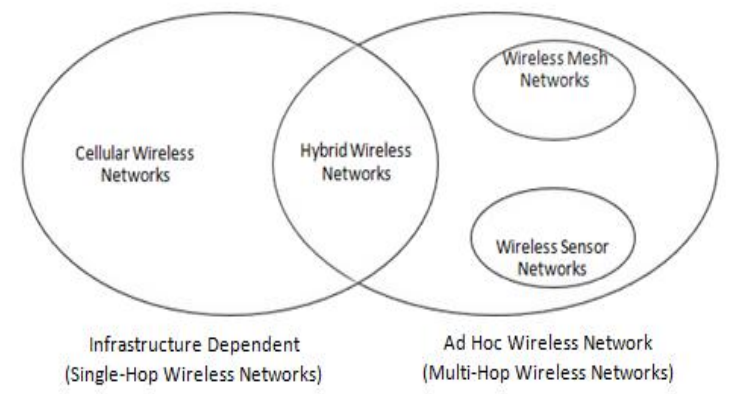

Fig 1: Cellular networks and ad hoc wireless networks

\subsection{Cellular systems}

Cellular systems [1] for mobile communications implement Space Division Multiplexing (SDM). Each base station (a.k.a transmitter) covers a certain area i.e. a cell. The radius of a cell can vary from tens of meters in buildings, and hundreds of meters in cities, up to tens of kilometers in the countryside. The shape of cells are never perfect circles or hexagons, but the shape depend on the environment (like buildings, mountains, valleys etc.), on climate situation, and seldom on the system load. A mobile telecommunication system is the typical example that uses this approach.

Here, a question arises "why mobile network providers install several thousands of base stations throughout a country (which is quite expensive) and do not use powerful transmitters with huge cells like, e.g., radio stations?"
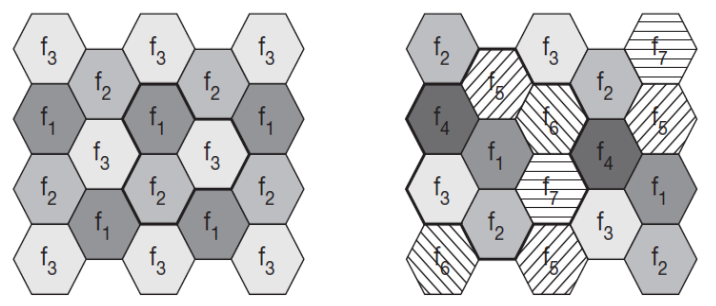

Fig 2: Cellular system with three and seven cell clusters

Thus, the above mention query can be responded by elaborating the advantages of cellular systems with small cells.

\subsubsection{Advantages of cellular systems with small cells are the following:}

- Higher capacity

Implementing SDM permits frequency reuse.

- Less transmission power
Power side is not a big problem for a base station; it is certainly problematic for a mobile station.

- Local interference only

With small cells, mobile stations and base stations only have to deal with 'local' interference.

- $\quad$ Robustness

Cellular systems are decentralized and so, they are more robust against the failure of single components. If one antenna collapses then communication gets affected only within a small area.

\subsubsection{Small cells also have some disadvantages that} are listed as below

- Infrastructure needed

Cellular systems require a complex infrastructure to connect the entire base stations. These incorporate many antennas, switches for call forwarding, location registers to find a mobile station etc; these all makes the entire system fairly costly.

- Handover needed

The mobile station has to perform a handover when moving from one cell to another cell. The handover happen quite often, depending on the cell size.

- $\quad$ Frequency planning

Frequencies have to be distributed carefully so as to avoid interference between base stations (transmitters) using same frequencies.

Finally, this chapter has presented the concept of cellular systems. Cellular systems implement SDM to raise the overall capacity of mobile phone systems. While these systems require detailed planning (i.e., cell size belonging the expected traffic), it gives the basic solutions for using the scarce (limited) frequency resources efficiently.

\subsection{Ad-Hoc Wireless Networks}

Ad-hoc wireless networks [1] [3] [6], however, do not require any infrastructure to work. Each node is capable of talking directly to other nodes, so access point controlling medium access is not required. Fig 3 shows two ad-hoc networks with three nodes each. Within an ad-hoc network nodes can only communicate, if they can reach each other physically, i.e., if they are present within radio range of each other or if other nodes forward the message. Nodes from the two networks shown in Fig 3 cannot, therefore, communicate with each other if they are not within the same radio range.
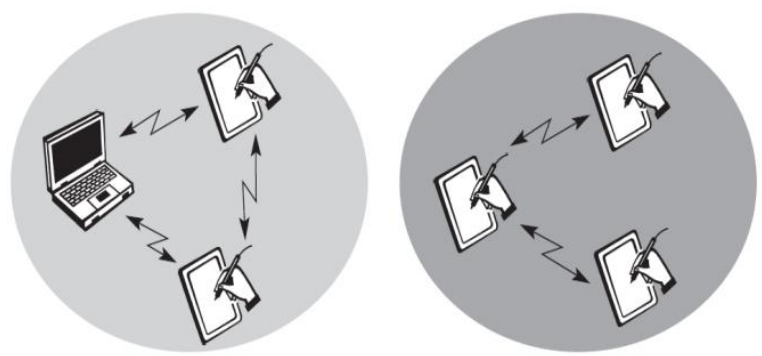

Fig 3: Example of two ad-hoc wireless networks

In ad-hoc networks, the complexity of each node is higher because every node has to implement medium access mechanisms and perhaps priority mechanisms, to handle hidden 
or exposed terminal problems and to provide a certain quality of service respectively. This type of wireless network shows the greatest possible flexibility in quick replacements of infrastructure or communication scenarios.

However, might be a selected nodes with the capabilities of forwarding data is present in an ad-hoc networks and most of the nodes have to connect to such that special node first in order to transmit data if the receiver is out of their range.

Single-hop [6] (All partners max. one hop apart), For Example: Bluetooth piconets, PDAs in a room, gaming devices.

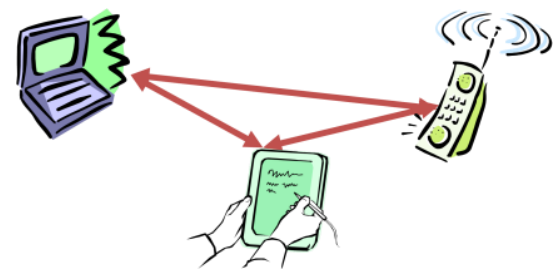

Fig 4: Single-hop

Multi-hop [6] (Cover larger distances, circumvents obstacles), For Example Bluetooth scatternet, TETRA police network, carto-car networks.

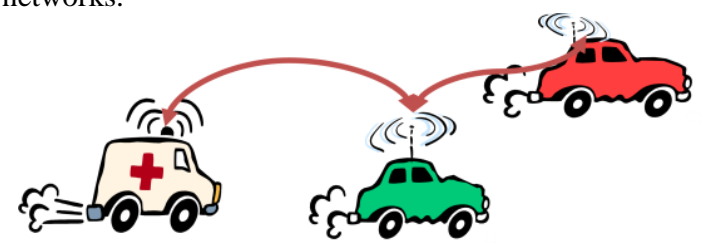

Fig 5: Multi-hop

Out of three WLANs: IEEE 802.11 and HiperLAN2 are usually needs infrastructure, and additionally they support ad-hoc networking. The third WLAN (Bluetooth) is the classical wireless ad-hoc network. Bluetooth focuses on spontaneous adhoc networks or on the simple connection of two or more devices without the setup of any infrastructure.

\subsection{Ad hoc Networks Characteristics}

\subsubsection{Mobility}

Nodes can be quickly repositioned. We can have individual random mobility, group mobility etc. The mobility model can have mainly significant force on the selection of a routing method and can thus influence the performance.

\subsubsection{Multihopping}

A multihop network is a network where the paths from source to destination pass through numerous nodes. Ad hoc nets frequently reveal multiple hops for obstacle negotiation, spectrum reuse and energy conservation.

\subsubsection{Self-organization}

The ad hoc network must unconventionally determine its own configuration parameters including addressing, routing, clustering, identification of position, power control etc.

\subsubsection{Energy conservation}

Most ad hoc nodes (e.g., laptops, PDAs, sensors, etc.) have limited power supply and no capability to generate their own power (e.g., solar panels). Energy efficient protocol design (e.g., MAC, routing, resource discovery, etc) is critical for longevity of the mission.

\subsubsection{Scalability}

In some applications (e.g., large environmental sensor fabrics, battlefield deployments, urban vehicle grids, etc) the ad hoc network can grow up to numerous thousand nodes.

\subsubsection{Security}

The ad hoc networks, however, are even more exposed to attacks than the infrastructure counterpart. Both active and passive attacks are possible. In active attack attacker tries to interrupt operations (control and data packets; reintroduces bogus control packets; damages the routing tables beyond repair; unleashes denial of service attacks, etc.). Passive attacks are unique in ad-hoc network and can be more hazardous than the active attack. The active attacker is eventually discovered and physically disabled. The passive attacker is never discovered by the network. It monitors data and control traffic patterns and thus infers the normal operation. Defence from passive attacks require powerful novel encryption techniques coupled with careful network protocol designs.

\subsubsection{Connection to the Internet}

As discussed, there is merit in extending the infrastructure wireless networks opportunistically with ad hoc appendices. The integration of ad hoc protocols with infrastructure standards is thus becoming a hot issue.

\subsection{Comparisons between Cellular and Ad- Hoc Wireless Networks}

Table 1. Comparison between Cellular and Ad-Hoc Wireless Networks

\begin{tabular}{|c|c|}
\hline Cellular Networks & Ad Hoc Wireless Networks \\
\hline Fixed infrastructure-based & Infrastructureless \\
\hline $\begin{array}{l}\text { Guaranteed bandwidth } \\
\text { (designed for voice traffic) }\end{array}$ & $\begin{array}{l}\text { Shared radio channel (more } \\
\text { suitable for best-effort data } \\
\text { traffic) }\end{array}$ \\
\hline Centralized routing & Distributed routing \\
\hline $\begin{array}{l}\text { Circuit-switched (evolving } \\
\text { toward packet switching) }\end{array}$ & $\begin{array}{l}\text { Packet-switched (evolving } \\
\text { toward emulation of circuit } \\
\text { switching) }\end{array}$ \\
\hline $\begin{array}{l}\text { Seamless connectivity (low } \\
\text { call drops during handoffs) }\end{array}$ & $\begin{array}{l}\text { Frequent path breaks due to } \\
\text { mobility }\end{array}$ \\
\hline $\begin{array}{l}\text { High cost and time of } \\
\text { deployment }\end{array}$ & $\begin{array}{l}\text { Quick and cost-effective } \\
\text { deployment }\end{array}$ \\
\hline $\begin{array}{l}\text { Reuse of frequency spectrum } \\
\text { through geographical } \\
\text { channel reuse }\end{array}$ & $\begin{array}{c}\text { Dynamic frequency reuse } \\
\text { based on carrier sense } \\
\text { mechanism }\end{array}$ \\
\hline $\begin{array}{l}\text { Easier to employ bandwidth } \\
\text { reservation }\end{array}$ & $\begin{array}{l}\text { Bandwidth reservation } \\
\text { requires complex medium } \\
\text { access control protocols }\end{array}$ \\
\hline $\begin{array}{l}\text { Application domains include } \\
\text { mainly civilian and } \\
\text { commercial sectors }\end{array}$ & $\begin{array}{l}\text { Application domains include } \\
\text { battlefields, emergency } \\
\text { search and rescue operations, } \\
\text { and collaborative computing }\end{array}$ \\
\hline $\begin{array}{l}\text { High cost of network } \\
\text { maintenance (backup power } \\
\text { source, staffing, etc.) }\end{array}$ & $\begin{array}{l}\text { Self-organization and } \\
\text { maintenance properties are } \\
\text { built into the network }\end{array}$ \\
\hline Mobile hosts are of relatively & Mobile hosts require more \\
\hline
\end{tabular}




\begin{tabular}{|c|c|}
\hline low complexity & $\begin{array}{c}\text { intelligence (should have a } \\
\text { transceiver as well as } \\
\text { routing/switching capability) }\end{array}$ \\
\hline $\begin{array}{c}\text { Major goals of routing and } \\
\text { call admission are to } \\
\text { maximize the call acceptance } \\
\text { ratio and minimize the call } \\
\text { drop ratio }\end{array}$ & $\begin{array}{c}\text { Main aim of routing is to } \\
\text { find paths with minimum } \\
\text { overhead and also quick } \\
\text { reconfiguration of broken } \\
\text { paths }\end{array}$ \\
\hline $\begin{array}{c}\text { Widely deployed and } \\
\text { currently in the third } \\
\text { generation of evolution }\end{array}$ & $\begin{array}{c}\text { Several issues are to be } \\
\text { addressed for successful } \\
\text { commercial deployment } \\
\text { even though widespread use } \\
\text { exists in defense }\end{array}$ \\
\hline
\end{tabular}

\section{APPLICATION OF AD HOC WIRELESS NETWORKS}

\subsection{Military applications}

An ad hoc wireless network is useful in establishing communication among a group of soldiers in a battle field.

\subsection{Collaborative and Distributed Computing}

A group of people in a conference can share data in ad hoc networks. Streaming of multimedia objects among the participating nodes.

\subsection{Emergency Operations}

Ad hoc wireless networks are functional in disaster operations such as searching operation and rescue operation and mob control.

\subsection{Wireless Mesh Networks}

A mesh networks which are built on wireless communications and allows for nonstop connections and reconfiguration around blocked paths by "hopping" from node to node until a connection can be established.

\subsection{Wireless Senor Networks}

Wireless Sensor Networks are a special category of ad hoc networks that are used to provide a wireless communication infrastructure among the sensors deployed in a specific application area. A wireless sensor network is a collection of a huge amount of sensor nodes that are organized in a particular area.

\subsection{Hybrid Wireless Networks}

One of the major application areas of ad hoc wireless networks is in hybrid wireless architectures such as multi-hop cellular networks (MCNs) and integrated cellular ad hoc (iCAR) networks. A multi-hop cellular network (MCNs) permits the communication through the base stations or multi-hop of mobile nodes. Integrated cellular ad hoc relay (iCAR) is a system that combines conventional cellular technology with Ad hoc Relay Station (ARS) technology together. In the resultant system cellular stations will communicate or redirect calls from the overcrowded cell to an adjacent one that is not crowded.

\section{ISSUES IN AD HOC WIRELESS NETWORKS}

There are various issues and challenges [1] while designing an ad hoc wireless system. The issues that affects the design, development and performance of an ad hoc wireless system are medium access scheme, addressing and service discovery (is essential because of absence of a centralized coordinator), Energy Management, Scalability, Self-Organization and
Security.

\section{ISSUES IN VARIOUS PROTOCOLS FOR AD-HOC WIRELESS NETWORKS 5.1 Issues in designing a MAC protocol for ad hoc wireless networks}

Since the characteristics of the wireless medium are completely different from those of the wired medium and ad hoc wireless networks need to deal with exclusive issues that are not applicable to wired networks. A diverse set of protocols is necessary for controlling access to the shared medium in such networks. Various Issues in designing a MAC protocol [5] for ad hoc wireless networks are listed as below:

\subsubsection{Bandwidth Efficiency}

Bandwidth efficiency can be defined as the ratio of the bandwidth used for actual data transmission to the total available bandwidth. As we know that nodes in ad hoc network share a common broadcast radio channel and the radio spectrum is limited, the bandwidth accessible for communication is also very limited. So the designing of MAC protocol must be done such a way that the scare bandwidth is utilized in an efficient manner.

\subsubsection{Quality of Service (QoS) Support}

QoS [4] support is necessary for supporting time-critical traffic sessions like time-critical traffic in military communications. MAC protocol for ad hoc wireless networks used in such realtime purpose must have some kind of a resource reservation mechanism that takes into consideration the nature of the wireless channel and the mobility of nodes.

\subsubsection{Synchronization}

Synchronization is very important for bandwidth reservations by nodes. The MAC protocol must take into consideration the synchronization between nodes in the network.

\subsubsection{Hidden and exposed problems}

The hidden and exposed problems are unique to wireless networks. The hidden terminal problem refers to the collision of packets at a receiving node due to the simultaneous transmission of those nodes that are not within the direct transmission range of the sender, but within the transmission range of the receiver.

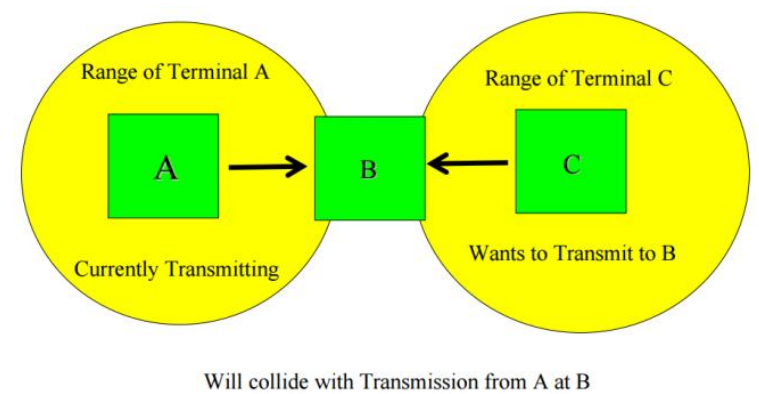

Fig 6: The hidden terminal problem [9]

Diagram before Shows the Scenario as: A sends to B, C cannot receive $\mathrm{A}, \mathrm{C}$ wants to send to $\mathrm{B}, \mathrm{C}$ senses a "free" medium (CS fails) collision at $\mathrm{B}, \mathrm{A}$ cannot receive the collision ( $\mathrm{CD}$ fails) $\mathrm{A}$ is "hidden" for $\mathrm{C}$.

The exposed terminal problem refers to the incapability of a node (blocked because of the transmission by nearby transmitting node) to pass on to another node. 


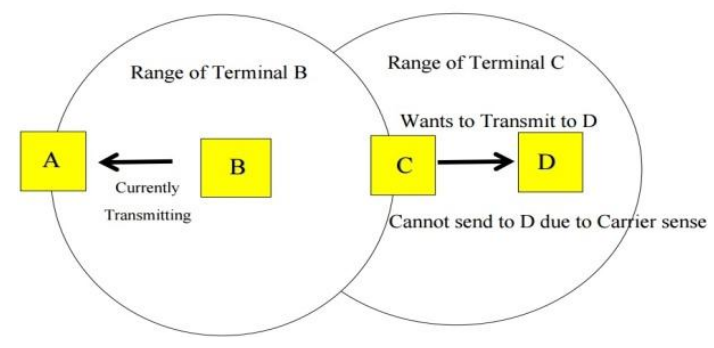

Fig 7: The exposed terminal problem [9]

\subsubsection{Distributed nature / lack of central coordination}

Ad hoc networks do not have centralized coordinators. In cellular networks, the base stations act as central coordinating nodes and allocate bandwidth to the mobile terminals. But this is not possible in an ad hoc network since all nodes remains moving frequently.

\subsubsection{Mobility of Nodes}

Nodes in an ad hoc wireless network are mobile most of the time. This is a very important factor affecting the performance (throughput) of the protocol. The protocol design must take this mobility factor into consideration so that the performance of the system is not significantly affected due to node mobility.

\subsection{Issues in designing a routing protocol for ad hoc wireless networks}

Routing protocols for Ad-Hoc Wireless Networks [8]: A variety of routing protocols for ad hoc wireless networks has been proposed in order to find a path to be followed by data packets from a source node to a destination node. Various Issues in designing a routing protocol for ad hoc wireless networks are:

\subsubsection{Mobility}

Wired network routing protocols cannot be used in ad hoc wireless networks where the mobility of the nodes results in frequently changing network topologies.

\subsubsection{Bandwidth constraint}

The limited bandwidth availability also imposes a constraint on routing protocols in maintaining the topological information. As efficient routing protocols in wired networks require the complete topology information, they may not be suitable for routing in the ad hoc wireless networking environment.

\subsubsection{Resource constraints}

Resource constraints form the major constraint for the nodes in an ad hoc wireless network are battery life and processing power since they are very essential and limited resources.

\section{OTHER AREA OF AD-HOC WIRELESS NETWORKS}

There are many more area in ad-hoc wireless networks which persists various issues and challenges in their designing and implementation. These areas are multicaste routing in ad-hoc wireless networks, transport layer and security protocols for adhoc wireless networks, Quality of Service (QoS) in ad-hoc wireless networks.

\section{CONCLUSION}

In this paper we have tried to survey ad-hoc wireless networks mainly from a technical point of view. We have also made an attempt to clarify what an ad hoc network actually is and what issues and challenges are faced by an ad hoc mobile wireless networks in finding a path between the communication end points of nodes that are mobile. In this paper we have also discussed the description of various routing methods for ad hoc wireless networks. The field of ad hoc wireless networks is rapidly rising and varying. Still many issues and challenges are existing in this field that may need to be addressed. It is usual to say that such networks will grow widespread use within the next few years. Furthermore there are various other wireless adhoc networks that are to be explored and introduced are Mobile ad hoc networks (MANET), Vehicular Ad hoc Networks (VANETs), Smart Phone Ad hoc Networks (SPANs), Internet based mobile ad hoc networks (iMANETs), Military / Tactical MANETs. Thus the mentioned areas can be taken as an open area for research and investigation.

\section{AUTHOR'S BIOGRAPHY}

Ms. Sushmita Kopekar is pursuing B.E. in ETC (Electronics \& Telecommunications Engineering) from G. H. Raisoni College of Engineering (An Autonomous Institution), Nagpur, INDIA. Currently she is studying in 7th Semester of B.E. Her main areas of interest are Mobile Communication and Wireless Networking.

Prof. Amresh Kumar completed M. Tech. in CSE (Computer Science \& Engineering) from Christ University, Bangalore, INDIA in 2013 and B.E. in Computer Technology from RTM Nagpur University, Nagpur, INDIA, in 2009. Currently, he is working as an assistant professor in the Department of Computer Technology in Rajiv Gandhi College of Engineering \& Research, Nagpur affiliated to RTMNU, INDIA. His main areas of interest are Big Data, Computer Network and Mobile Communication

\section{REFERENCES}

[1] C. Siva Ram Murthy \& B.S. Manoj, "Ad Hoc Wireless Networks Architectures and Protocols", Pearson Education, 2nd Edition, 2005.

[2] Amresh Kumar "A Survey on Current \& Traditional Routing Protocols for Ad Hoc Wireless Networks", IJCSNS International Journal of Computer Science and Network Security, VOL.13 No.10, October 2013.

[3] Chakrabarti, "Quality of Service in Mobile Ad Hoc Networks", Handbook of Ad Hoc Wireless Networks, CRC press, 2003.

[4] Sunita Prasad and Zaheeruddin, "A REVIEW AND COMPARISON OF QUALITY OF SERVICE ROUTING IN WIRELESS AD HOC NETWORKS", International Journal of Wireless \& Mobile Networks (IJWMN) Vol. 3, No. 1, February 2011.

[5] Elizabeth M. Royer, Chai-Keong Toh, "A Review of Current Routing Protocols for Ad Hoc Mobile Wireless Networks", IEEE Personal Communications, April 1999.

[6] http://en.wikipedia.org/wiki/Wireless_ad_hoc_network.

[7] http://www.ijarcsse.com/docs/papers/Volume_3/6_June201 3/V3I6-0379.pdf

[8] http://ieeexplore.ieee.org/xpl/articleDetails.jsp?arnumber=1 385906.

[9] http://www.cs.jhu.edu/ cs647/intro_adhoc.pdf. 